56, 3, pp. 793-802, Warsaw 2018

DOI: $10.15632 /$ jtam-pl.56.3.793

\title{
MATHEMATICAL MODEL OF LEVITATING CART OF MAGNETIC UAV CATAPULT
}

\author{
Anna Sibilska-Mroziewicz, Edyta Ładyżyńska-Kozdraś \\ Warsaw University of Technology, Faculty of Mechatronics, Warsaw, Poland \\ e-mail: a.sibilska@mchtr.pw.edu.pl; e.ladyzynska@mchtr.pw.edu.pl
}

\begin{abstract}
The article presents the steps of modeling of the dynamics of a levitating cart of an unmanned aerial vehicle (UAV) magnetic catapult. The presented in the article innovative catapult is based on the Meissner effect occurring between high-temperature superconductors (HTS) and a magnetic field source. The catapult suspension system consists of two elements: fixed to the ground base with magnetic rails and a moving cart. Generating magnetic field rails are made of neodymium magnets. Levitation of the launcher cart is caused by sixteen superconductors YBCO, placed in the cart frame supports. Described in the article model contains the system of Cartesian reference frames, kinematic constrains, equations of motion and description of forces acting on the cart as well as exemplary numerical simulation results.
\end{abstract}

Keywords: magnetic catapult, equations of motion, Meissner effect

\section{Introduction}

The growing demand for commercial unmanned aerial vehicles (UAV) requires exploration of innovative technical solutions associated with critical aspects of the use of such facilities. Safe procedures for UAV take-off and landing are one of such issues. Most of unmanned aerial vehicles have neither sufficient power supply nor a structure for self-start, especially in the field of uneven ground and insufficient runway.

Only a few of UAVs such as Predator have a chassis system allowing for self-start and landing. The chassis system, however, increases mass of the vehicle, makes its construction more complicated and requires implementation of advanced algorithms of control during take-off and landing. Moreover, the take-off procedure itself requires significant reserves of power. Take-off of an unmanned aerial vehicle can also be done by throwing it from a human hand. However, this way is only possible in the case of small and lightweight UAVs such as Raven used by the US Army.

In other cases it is necessary to use a separate device called a launcher or airplane catapult. Nowadays, it is common to use rocket systems (RATO-Rocket Assisted Take Off), bungee cord, hydraulic and pneumatic launchers (Fahlstrom and Gleason, 2012). An attractive alternative to current systems are magnetic catapults. Magnetic catapults compared with classical solutions enable safe, non-impact service of the UAV launch process and allow achieving much bigger final UAV speed. NASA plans using electrodynamics catapults to launch spacecraft (Polzin et al., 2013) and hypersonic planes. Magnetic catapults are also planned to replace steam catapults used on aircraft carriers (Bertoncelli et al., 2002).

Nowadays, magnetic suspension systems are used in high-speed trains (Mag-Lev). Currently, two types of Mag-Lev technology are developed commercially (Liu et al., 2015). Electromagnetic suspension (EMS) developed by the German Transrapid system. The suspension is based on the strength attraction force between metal rails and mounted on the underside of the train 
electromagnets. Built in 2004 Shanghai Maglev train uses the EMS technology. The second solution is an electrodynamic suspension system (EDS) proposed by Central Japan Railway Company. The EDS system uses strong repulsive forces generated by strong superconducting electromagnets built into the train path and trains chassis. Built in Japan Chuo Shinkansen line, which is the fastest train in the world (Coates, 2007), is based on the EDS technology. An alternative to EMS and EDS systems are passive suspensions using diamagnets.

This paper presents a mathematical model of a new UAV catapult prototype based on "out of the box" idea, using HTS in the launcher suspension system. According to the Meissner effect, superconductors cooled down below the critical temperature shield the external magnetic field generated by permanent magnets or electromagnets. The Meissner effect ensures spectacular levitation of the superconductor above the source of the magnetic field (Fig. 1).
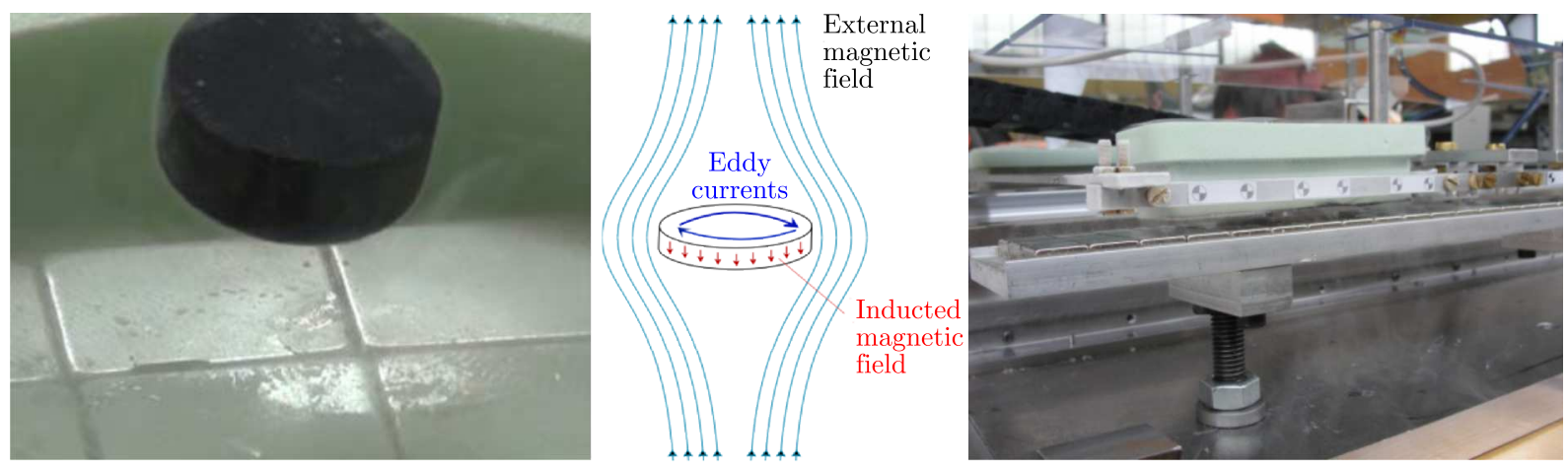

Fig. 1. Meissner effect

The initial part of the article presents a prototype of a magnetic UAV catapult using the Meissner effect. The following considerations include itemized assumptions regarding physical model and description of the system of Cartesian coordinate frames. Subsequent discussion contains equations of motion of the levitating cart and description of kinematic constrains and loads acting on the cart. The considerations are closed by results of numerical simulations.

\section{Catapult prototype}

Figure 2 shows photographs of the prototype of a magnetic launcher designed within FP7, EU GABRIEL (Integrated Ground and on-board system for Support of the Aircraft Safe Take-off and Landing) (Rohacs, 2015), (Falkowski, 2016), (Falkowski, Sibilski, 2013). During the take-off procedure, the UAV is attached to the levitating cart which is a movable part of the launcher. The cart is driven by a linear motor. The construction of the cart consists of a rigid frame made of duralumin and four containers. In each container, there are four high-temperature superconductors YBCO with a critical temperature of $92 \mathrm{~K}$. These superconductors have a cylindrical shape with diameter of $21 \mathrm{~mm}$ and height of $8 \mathrm{~mm}$. After filling the containers with liquid nitrogen, superconductors transit into superconducting state and start hovering over the launcher tracks due to the Meissner phenomena. Levitation phenomenon ends when the YBCO temperature exceeds the critical temperature of $92 \mathrm{~K}$. Therefore, the material of the container should provide the maximum thermal isolation. The starting path, mounted to the launcher base, consists of two parallel rails, each made of three rows of permanent magnets. To build the prototype, rectangular neodymium magnets polarized top-down have been used.

Potential use of passive suspensions, based on HTS in transportation systems, would bring many benefits. The levitation phenomenon enables frictionless longitudinal movement of the cart. The levitation gap remains stable without any feedback loop, and low complexity of the system would improve it is reliability. The biggest defect of the solution is the need of cooling 
down and maintaining superconductors in low temperatures. Systems which use passive magnetic suspension, despite growing popularity, have not been used so far in professional and commercial technical solutions. However, there is a small number of academic research projects on the use of HTS in transportation systems, including SupraTrans (Schultz et al., 2005), Cobra Tram (Sotelo et al., 2011) and SuperMaglev (Wang et al., 2005).
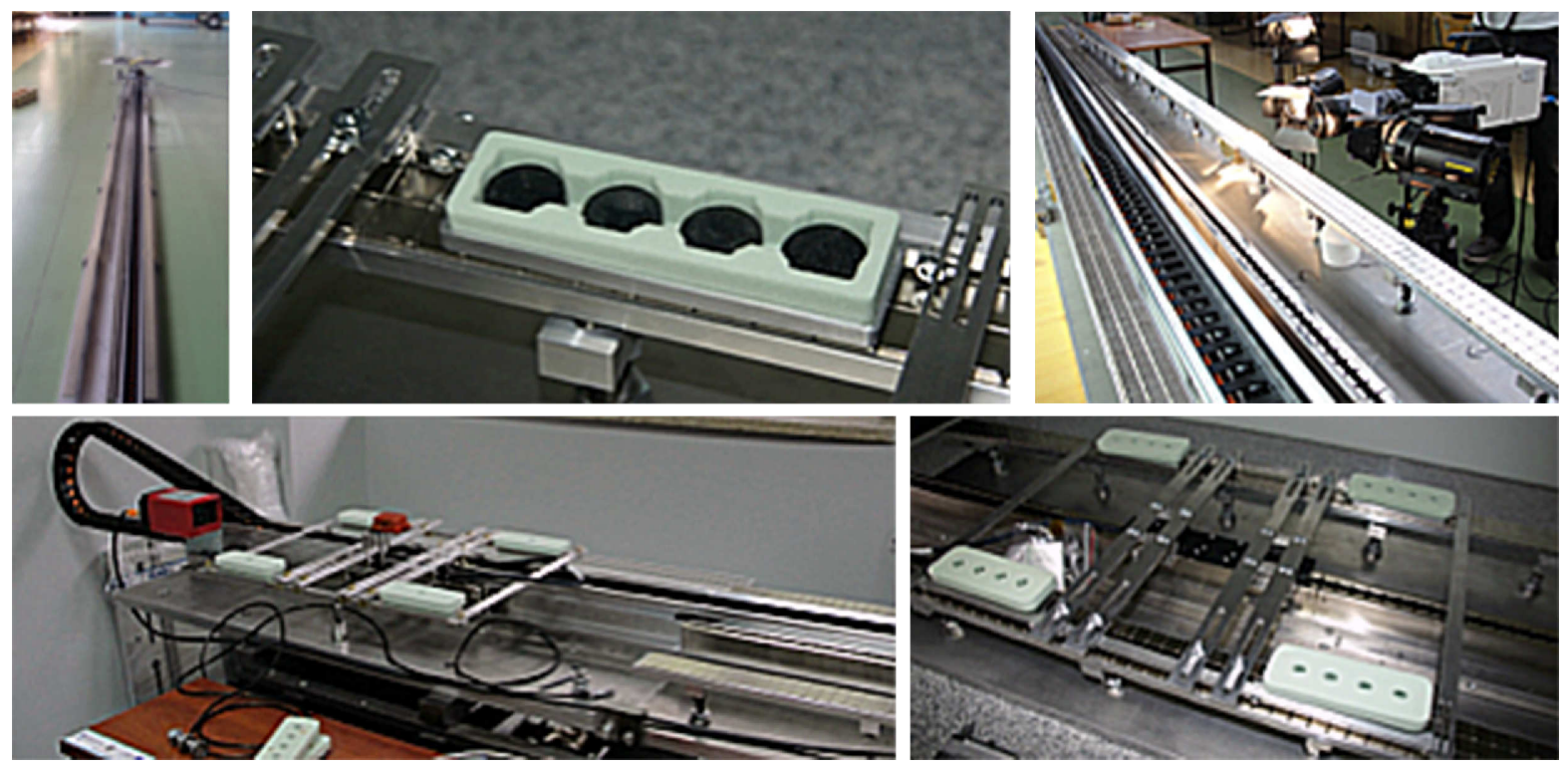

Fig. 2. Prototype of a magnetic UAV catapult using the Meissner effect

\section{Physical model}

An extremely important step in the modeling procedure (Eadyżyńska-Kozdraś, 2011) is definition of the physical model being the basis of formulation of the mathematical model. The proposed physical model of the levitating cart assumes the following simplifications:

- the levitating cart is an axisymmetric solid body with six degrees of freedom;

- mass and center of mass of the cart do not change during movement, however, the position of the taking off airplane may change;

- the system motion is considered only in a no wind environment;

- motion of the cart is controlled in only one direction by a linear motor;

- the cart is levitating above magnetic rails due to the Meissner effect;

- cart movement results from gravitational, magnetic and propulsion forces acting on the cart itself and gravitational, aerodynamical and propulsion forces acting on the taking off airplane;

- mass of evaporating nitrogen is not considered.

\section{Reference frames}

In order to describe the dynamics of the levitating cart, the following clockwise Cartesian coordinates frames are attached to the following parts of the UAV magnetic launcher:

- The motionless base system $O x_{f} y_{f} z_{f}$ is a rectangular Cartesian coordinate system rigidly connected with the ground. The $O z_{f}$ axis is directed vertically downward, in the direction of gravitational acceleration; the $O x_{f}$ axis coincides with the horizontal projection of the 
aircraft taking-off path; the $O y_{f}$ axis completes the right-handed coordinate system. The base frame origin coincides with the starting point of the catapult cart. The position of the levitating cart, along the $O x_{f}$ frame, could be measured by a laser sensor.

- The magnetic reference frame $O x_{m} y_{m} z_{m}$ is a coordinate system in which magnetic interactions and cart propulsion forces are modeled. The $O x_{m}$ axis covers the catapult axis of symmetry; the $O z_{m}$ axis points down perpendicularly to the catapult base; the $O y_{m}$ axis connects the left and right catapult rails. The system origin is moving along the catapult axis of symmetry $O x_{m}$ and covers the projection of the levitating cart center of mass into the $O x_{m}$ axis. Orientation of the magnetic reference frame, due to the fixed system $O x_{f} y_{f} z_{f}$, is described by two angles $\theta_{m / f}$ and $\phi_{m / f}$. The angle $\theta_{m / f}$ corresponds to deliberate inclination of the catapult base, relative to the horizontal plane. A non zero value of the $\phi_{m / f}$ angle is caused by imbalances of the substrate on which the launcher is placed.

- The $O x_{s} y_{s} z_{s}$ coordinate system describes the position and orientation of the levitating cart. The origin of the cart coordinate system is fixed with the cart center of mass, and its axes are rigidly connected with the cart frame. The $O x_{s}$ axis points along the longitudinal cart dimension; the $O z_{s}$ axis is perpendicular to the cart surface and points downward; the $O y_{s}$ axis is parallel to the lateral cart dimension and completes the right-handed coordinate system. Orientation of the launcher cart with respect to the magnetic coordinate system is described by three qusi-Euler angles $\theta_{s / m}, \psi_{s / m}$ and $\phi_{s / m}$.

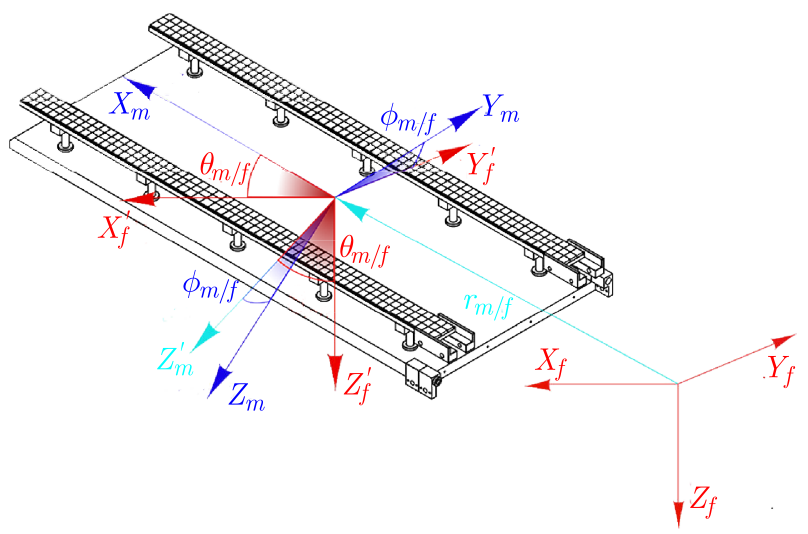

Fig. 3. Coordinate systems fixed with the magnetic launcher: inertial $O x_{f} y_{f} z_{f}$ and magnetic $O x_{m} y_{m} z_{m}$

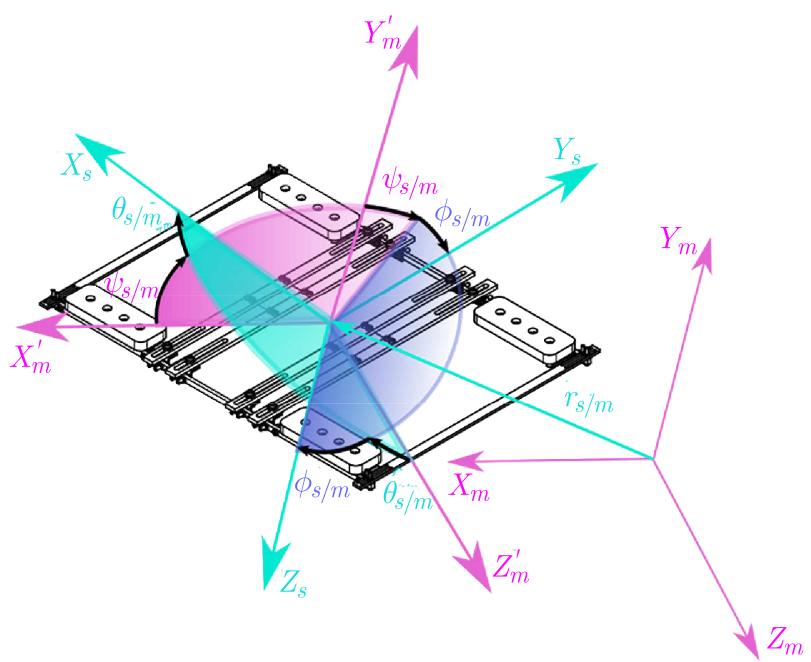

Fig. 4. The coordinate system fixed with the levitating cart $O x_{s} y_{s} z_{s}$ 
- The axes of the gravitational reference frame $O x_{g} y_{g} z_{g}$ are parallel to the inertial frame $O x_{f} y_{f} z_{f}$. The system origin is fixed with the cart center of mass. In that system, the gravitational forces and torques are described.

- The reaction forces and torques acting between the cart and taking-off airplane are described in the $O x_{c} y_{c} z_{c}$ coordinate system. The system origin and its orientation is dictated by the way of attaching the UAV into the cart frame.

\section{Kinematic constrains}

Motion of the cart is described by time and space coordinates located in the event space. The cart position, at the particular moment, is unambiguously described by linear and angular coordinates and velocities. Those coordinates change with time and are coupled by kinematic constrains. It is important to maintain a mutual coordinate system while describing the function of particular coordinates. Delivered below equations describing cart kinematic constrains are described in the $O x_{s} y_{s} z_{s}$ system fixed with the cart center of mass.

The vector of the current cart position in the fixed to the ground inertial frame $O x_{f} y_{f} z_{f}$ is described by

$$
\mathbf{r}=x_{f} \mathbf{i}_{f}+y_{f} \mathbf{j}_{f}+z_{f} \mathbf{k}_{f}
$$

The vector of instantaneous linear velocity $\mathbf{V}$ described in the frame fixed with the cart frame $O x_{s} y_{s} z_{s}$ has three components: longitudinal $U$, lateral $V$ and climb speed $W$

$$
\mathbf{V}=U \mathbf{i}_{s}+V \mathbf{j}_{s}+W \mathbf{k}_{s}
$$

The vector of instantaneous angular velocity $\Omega$ described in the fixed to the cart frame system $O x_{s} y_{s} z_{s}$ has the following components: roll rate $P$, pitch rate $Q$ and yaw rate $R$

$$
\boldsymbol{\Omega}=P \mathbf{i}_{s}+Q \mathbf{j}_{s}+R \mathbf{k}_{s}
$$

Kinematic constraints between linear velocity components measured relative to the inertial coordinate system $O x_{f} y_{f} z_{f}$, and linear velocities $U, V, W$ measured relative to the coordinate system $O x_{s} y_{s} z_{s}$, fixed to the cart, have the following form

$$
\left[\begin{array}{c}
U \\
V \\
W)
\end{array}\right]=R_{s / m} R_{m / f}\left[\begin{array}{c}
\dot{x}_{f} \\
\dot{y}_{f} \\
\dot{z}_{f}
\end{array}\right]
$$

The rotation matrices $\mathbf{R}_{s / m}$ and $\mathbf{R}_{m / f}$ are described by equations (5.5) with notations: $c \alpha=\cos \alpha$ and $s \alpha=\sin \alpha$

$$
\begin{aligned}
\mathbf{R}_{s / m}= & {\left[\begin{array}{ccr}
c \theta_{s / m} c \psi_{s / m} & c \theta_{s / m} s \psi_{s / m} & -s \theta_{s / m} \\
s \phi_{s / m} s \theta_{s / m} c \psi_{s / m}-c \phi_{s / m} s \psi_{s / m} & s \phi_{s / m} s \theta_{s / m} s \psi_{s / m}+c \phi_{s / m} c \psi_{s / m} & s \phi_{s / m} c \theta_{s / m} \\
c \phi_{s / m} s \theta_{s / m} c \psi_{s / m}+s \phi_{s / m} s \psi_{s / m} & c \phi_{s / m} s \theta_{s / m} s \psi_{s / m}-s \phi_{s / m} c \psi_{s / m} & c \phi_{s / m} c \theta_{s / m}
\end{array}\right] } \\
\mathbf{R}_{m / f}= & {\left[\begin{array}{ccc}
c \theta_{s / m} & 0 & -s \theta_{s / m} \\
s \phi_{s / m} s \theta_{s / m} & c \phi_{s / m} & s \phi_{s / m} c \theta_{s / m} \\
c \phi_{s / m} s \theta_{s / m} & -s \phi_{s / m} & c \phi_{s / m} c \theta_{s / m}
\end{array}\right] }
\end{aligned}
$$

The components of instantaneous angular velocities $P, Q, R$ are combinations of generalized velocities $\dot{\theta}_{s / m}, \dot{\psi}_{s / m}, \dot{\phi}_{s / m}$ and trigonometric functions of angles: $\theta_{s / m}, \psi_{s / m}$ and $\phi_{s / m}$, according to the relation

$$
\left[\begin{array}{l}
P \\
Q \\
R
\end{array}\right]=\left[\begin{array}{ccc}
1 & 0 & -\sin \theta_{s / m} \\
0 & \cos \phi_{s / m} & \sin \phi_{s / m} \cos \theta_{s / m} \\
0 & -\sin \phi_{s / m} & \cos \phi_{s / m} \cos \theta_{s / m}
\end{array}\right]\left[\begin{array}{c}
\dot{\phi}_{s / m} \\
\dot{\theta}_{s / m} \\
\dot{\psi}_{s / m}
\end{array}\right]
$$




\section{Equations of motion}

To derive equations of motion for objects treated as rigid bodies, mostly Newtonian approach is used, i.e. forces, momentum and angular momentum conservation laws. Examples could be found in (Baranowski, 2016). Sometimes, more involved theoretical mechanics is used, e.g. Lagrangian formulation, see (Koruba et al., 2010), Boltzmann-Hamel equations like in (Ładyżyńska-Kozdraś and Koruba, 2012) or Maggi equations (Eadyżyńska-Kozdraś, 2012).

In the presented consideration, the levitating cart is treated as a rigid body with six degrees of freedom. The proposed equations of motions are described in the coordinate frame fixed to the cart center of mass $O x_{s} y_{s} z_{s}$. The presented mathematical model is developed according to principal mechanical laws: the momentum and angular momentum conservation principles.

- The derivative of the momentum $\Pi$ with respect to time

$$
\frac{\partial \boldsymbol{\Pi}}{\partial t}+\boldsymbol{\Omega} \times \mathbf{\Pi}=\mathbf{F}
$$

- The derivative of the angular momentum $\mathbf{K}$ with respect to time

$$
\frac{\partial \mathbf{K}}{\partial t}+\boldsymbol{\Omega} \times \mathbf{K}+\mathbf{V} \times \mathbf{\Pi}=\mathbf{M}
$$

The principle of conservation of the momentum and angular momentum can be applied to the problem in two ways. Firstly, the levitating cart and taking-off airplane are treated as a single undivided rigid body. The momentum and angular momentum of that body is defined for the entire object, relative to the one arbitrarily chosen pole which does not necessarily coincide with the center of mass. The second way is to designate moments and angular moments separately for the taking off plane and the levitating cart. The presented equations consider both movable parts of the catapult as a one rigid body.

The general form of equations of motion of the cart in the three dimensional space is expressed by the relationship (Ładyżyńska-Kozdraś, 2011)

$$
\mathbf{M} \dot{\mathbf{V}}+\mathbf{K M V}=\mathbf{Q}
$$

where:

- matrix of inertia

$$
\mathbf{M}=\left[\begin{array}{ccc|ccc}
m & 0 & 0 & 0 & S_{z} & -S_{y} \\
0 & m & 0 & -S_{z} & 0 & S_{z} \\
0 & 0 & m & S_{y} & -S_{x} & 0 \\
\hline 0 & -S_{z} & S_{y} & I_{x} & -I_{x y} & -I_{x z} \\
S_{z} & 0 & -S_{x} & -I_{y x} & I_{y} & -I_{y z} \\
-S_{y} & S_{x} & 0 & -I_{z x} & -I_{z y} & I_{z}
\end{array}\right]
$$

- matrix of kinematic constrains

$$
\mathbf{K}=\left[\begin{array}{ccc|ccc}
0 & -R & Q & 0 & 0 & 0 \\
R & 0 & -P & 0 & 0 & 0 \\
-Q & P & 0 & 0 & 0 & 0 \\
\hline 0 & -W & V & 0 & -R & Q \\
W & 0 & -U & R & 0 & -P \\
-V & U & 0 & -Q & P & 0
\end{array}\right]
$$

- vector of linear and angular accelerations

$$
\dot{\mathbf{V}}=[\dot{U}, \dot{V}, \dot{W}, \dot{P}, \dot{Q}, \dot{R}]^{\mathrm{T}}
$$


— vector of linear and angular velocities

$$
\mathbf{V}=[U, V, W, P, Q, R]^{\mathrm{T}}
$$

— vector of external forces and torques

$$
\mathbf{Q}=\left[\begin{array}{c}
\mathbf{F} \\
\mathbf{M}
\end{array}\right]
$$

After determining kinematic constrains as well as forces $\mathbf{F}$ and torques $\mathbf{M}$ acting on the levitating cart, a general model of the system dynamics is obtained.

\section{Forces and torques acting on levitating cart}

Forces and torques acting on the considered system results from interactions between the taking-off UAV, levitating cart and the generated by the catapult rails magnetic field. The vector of external forces and torques $\mathbf{Q}$ can be described by superposition of the vectors of forces and torques: acting only on the cart frame $\mathbf{Q}^{S}$, acting on the UAV alone $\mathbf{Q}^{B}$, and describing the method of attachment of the taking-off UAV into the cart frame

$$
\mathbf{Q}=\mathbf{Q}^{S}+\mathbf{Q}^{B}+\mathbf{Q}^{S B}
$$

The cart frame undergoes the gravitational pull $\mathbf{Q}_{g}^{S}$, propulsion forces $\mathbf{Q}_{T}^{S}$, aerodynamic interactions $\mathbf{Q}_{A}^{S}$ and the load resulting from the Meissner effect, called levitation forces $\mathbf{Q}_{L_{i}}^{S}$. The levitation forces depend on the position of the box with superconductors relative to the rails generating magnetic field. This distance is called the levitation gap. Taking into account changes in the orientation of the cart, the levitation forces $Q_{L_{i}}^{S}$ are determined separately for each of four containers with superconductors

$$
\mathbf{Q}^{S}=\mathbf{Q}_{g}^{S}+\mathbf{Q}_{T}^{S}++\mathbf{Q}_{A}^{S} \sum_{i=1}^{4} \mathbf{Q}_{L_{i}}^{S}
$$

The taking-off UAV moves under the influence of the gravitational pull $\mathbf{Q}_{g}^{B}$, propulsion forces $\mathbf{Q}_{T}^{B}$, aerodynamic interactions $\mathbf{Q}_{A}^{B}$ and the load resulting from the UAV control system $\mathbf{Q}_{\delta}^{B}$

$$
\mathbf{Q}^{B}=\mathbf{Q}_{g}^{B}+\mathbf{Q}_{T}^{B}+\mathbf{Q}_{A}^{B}+\mathbf{Q}_{\delta}^{B}
$$

The loads $\mathbf{Q}_{g}^{S}$ and $\mathbf{Q}_{g}^{B}$ are described in the gravitational system $O x_{g} y_{g} z_{g}$. The linear drive propulsion forces $\mathbf{Q}_{T}^{S}$ and levitation forces $\mathbf{Q}_{L_{i}}^{S}$ are considered in relation to the magnetic system $O x_{m} y_{m} z_{m}$. Therefore, it is necessary to transform the individual vectors to the coordinate system attached to the levitating cart, by multiplying them by the corresponding transformation matrix. The value of the nonlinear levitation force depends on the gap between the cart support and magnetic rails. The smaller the gap, the greater the force. The levitation force is modeled as a concentrated force acting on the center of the box with superconductors.

\section{Preliminary numerical simulation}

The use of the momentum and angular momentum laws of conservation for mechanical systems makes it possible to develop the dynamical model of the take-off of an unmanned aircraft. 
During the take-off procedure, the UAV is attached to the levitating cart, which is a movable part of the launcher. In the analyzis, the UAV class micro Bell 540 has been taken into account. In the research, it is assumed that the linear-driven cart moves with constant horizontal acceleration.

The presented in the article mathematical model of the levitating cart of the magnetic UAV catapult is the theoretical basis for preliminary numerical simulations. The obtained numerical results show correctness of the developed mathematical model. As shown in Figs. 5 and 6, the unmanned aircraft take-off takes place in a proper manner. It maintains the preset parameters resulting from the adopted guidance parameters of the levitating cart of the magnetic UAV catapult.

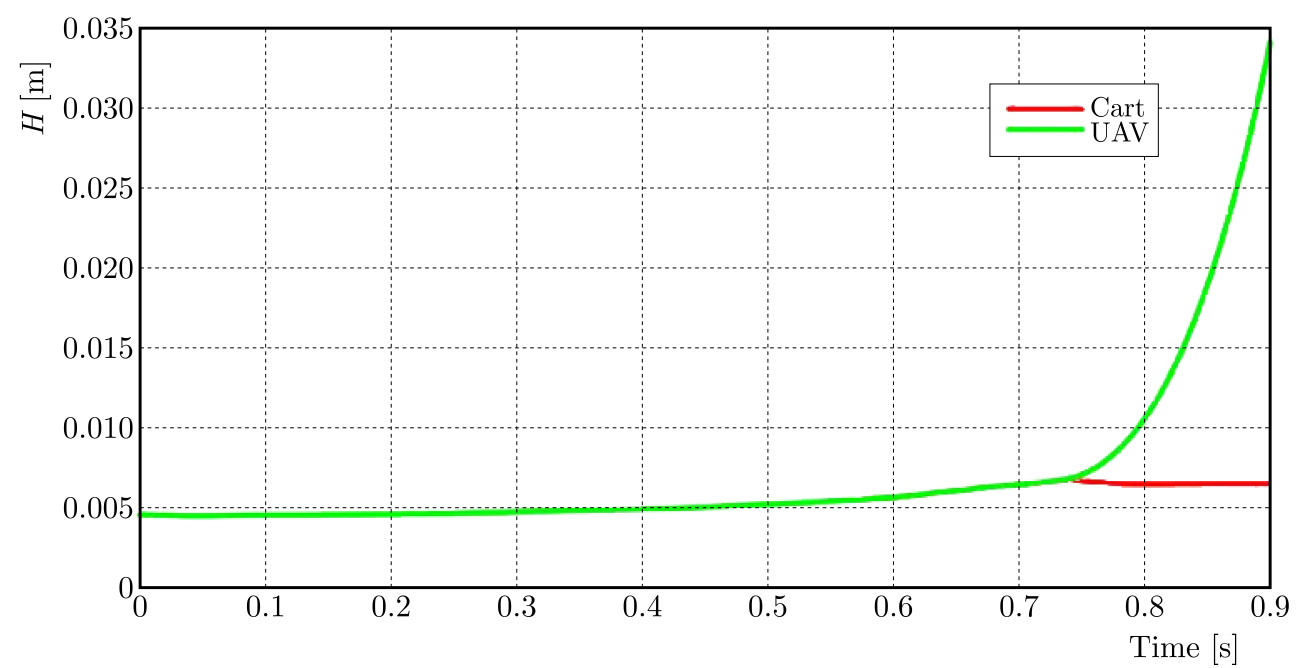

Fig. 5. The course of changes in height of the levitating cart and UAV at the moment of take-off

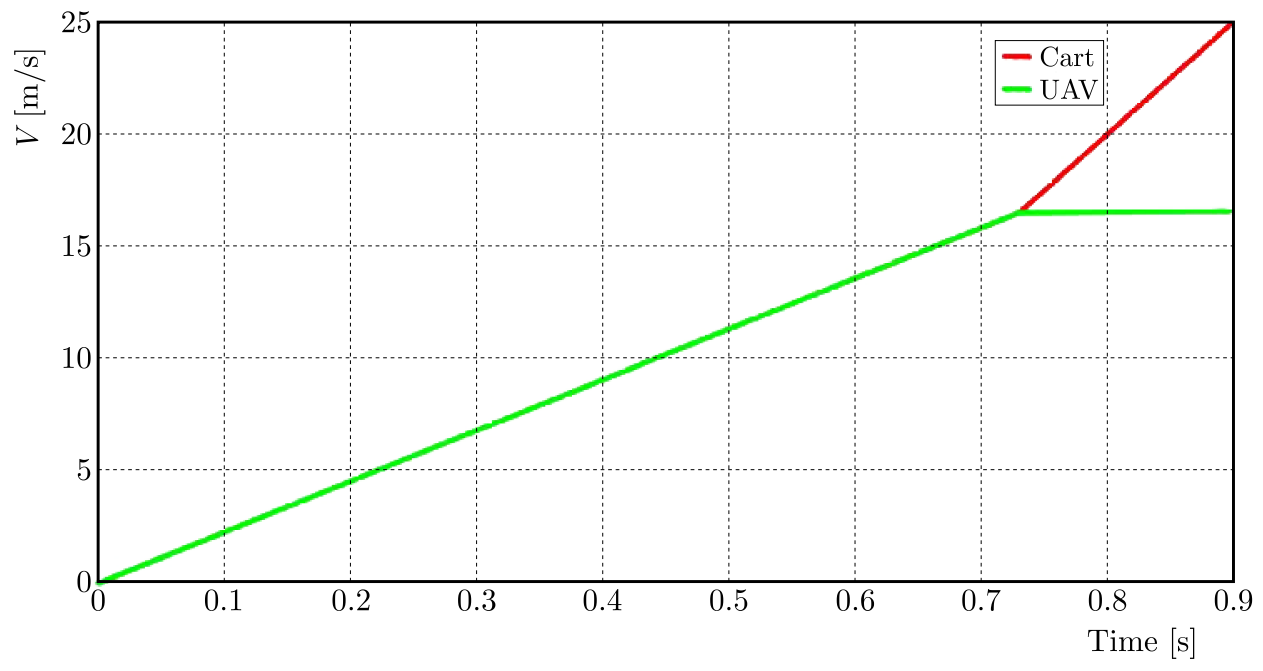

Fig. 6. Velocity of the levitating cart and UAV at the moment of take-off

\section{Conclusions}

The paper is addressing an interesting subject related to dynamics and modelling methodology of the levitating cart of a magnetic UAV catapult. The presented mathematical model has been developed according to principal mechanical laws - the momentum and angular momentum of conservation. The considerations are closed by exemplary numerical results. 
Described work is a prelude to wider research on the mechanical properties of the launcher levitating cart, being under the influence of forces, generated by levitation of HTS in the magnetic field.

The presented in this article model does not take into consideration the non-uniformness of magnetic field and the flux pinning effect occurring in II type superconductors. Description of levitation forces and torques, based on both theoretical investigations and laboratory tests, will be the next step of research as well as development of a more general mathematical model including UAV dynamics and non-uniformness of the magnetic field distribution.

\section{References}

1. Baranowski L., 2016, Explicit "ballistic M-model": a refinement of the implicit "modified point mass trajectory model", Bulletin of the Polish Academy of Sciences Technical Sciences, 64, 1

2. Bertoncelli T., Monti A., Patterson D., Dougal R., 2002, Design and simulation of an electromagnetic aircraft launch system, Power Electronics Specialists Conference, 2002. pesc 02. 2002 IEEE 33rd Annual, 3, 1475-1480

3. Coates K.C., 2007, TGV's 357Mph Demo Proves HSM's Superiority, North American Maglev Transport Institute

4. Fahlstrom P., Gleason T., 2012, Introduction to UAV Systems, Wiley, Aerospace Series, ISBN 978-11-1839-681-0

5. Falkowski K., 2016, Passive Magnetic Suspension (in Polish), Military University of Technology, ISBN 978-83-7938-115-9

6. FALKOWSKi K., Huścio T., 2009, Modelling of the magnetic attraction force of the electromagnetic module in the relative base - air-gap - absolute base system, Solid State Phenomena, 144, 53-58

7. Falkowski K., SiBiLski K., 2013, Magnetic levitation system for take-off and landing airplane project GABRIEL, COMSOL Conference 2013, Rotterdam

8. Koruba Z., Dziopa Z., Krzysztofik I., 2010, Dynamics and control of a gyroscope-stabilized platform in a self-propelled anti-aircraft system, Journal of Theoretical and Applied Mechanics, 48, $1,5-26$

9. Lichota P., Sibilski K., Ohme P., 2016, D-optimal simultaneous multistep excitations for aircraft parameter estimation, Journal of Aircraft, http://dx.doi.org/10.2514/1.C033794

10. Liu Z., Long Z., Li X., 2015, MagLev Trains: Key Underlying Technologies, Springer Tracts in Mechanical Engineering, ISBN 978-3-662-45673-6

11. ŁADYŻYŃSKA-KOZDRAŚ E., 2011, The modelling and numerical simulation of the controlled, movable objects with imposed non-holonomic constraints treated as control laws (in Polish), Mechanika, 237, Oficyna Wydawnicza Politechniki Warszawskiej

12. ŁADYŻYŃSKA-KOZDRAŚ E., 2012, Modeling and numerical simulation of unmanned aircraft vehicle restricted by non-holonomic constraints, Journal of Theoretical and Applied Mechanics, 50, 1, $251-268$

13. ŁadyżyŃSka-Kozdraś E., Koruba Z., 2012, Model of the final section of navigation of a self-guided missile steered by a gyroscope, Journal of Theoretical and Applied Mechanics, 50, 2, $473-485$

14. Polzin K.A., Adwar J.E., Hallock A.K., 2013, Optimization of electrodynamic energy transfer in coilguns with multiple, uncoupled stages, IEEE Transactions on Magnetics, 49, 4, 1453-1460

15. Rohacs D., Rohacs J., 2015, Magnetic levitation assisted aircraft take-off and landing (feasibility study - GABRIEL concept), Progress in Aerospace Sciences, ISSN 0376-0421 
16. Schultz L., de HaAs O., Verges P., Beyer C., Rohlig S., Olsen H., Kuhn L., Berger D., Noteboom U., Funk U., 2005, Superconductively levitated transport system - the SupraTrans project, IEEE Transactions on Applied Superconductivity, 15, 2, 2301-2305

17. Sotelo G.G., Dias D.H.N., De Andrade R., Stephan R.M., 2011, Tests on a superconductor linear magnetic bearing of a full-scale MagLev vehicle, IEEE Transactions on Applied Superconductivity, 21, 1464-1468

18. Wang J., Wang S., Deng C., Zeng Y., Song H., Huang H., 2005, A superhigh speed has MagLev vehicle, International Journal of Modern Physics B, 19, 01n03, 399-401

Manuscript received July 10, 2016; accepted for print December 28, 2017 